\title{
Discovery of pulsation in a helium-rich subdwarf B star ${ }^{\star}$
}

\author{
A. Ahmad and C. S. Jeffery \\ Armagh Observatory, College Hill, Armagh BT61 9DG, N. Ireland, UK \\ e-mail: amir@star.arm.ac.uk \\ Received 7 January 2005 / Accepted 1 June 2005

\begin{abstract}
Helium-rich subdwarf B (He-sdB) stars form a very small inhomogeneous group of subluminous stars showing varying degrees of helium enrichment. They have been found in the field of our galaxy as well as in globular clusters. Here we report the first discovery of pulsation in a He-sdB star - LS IV $-14^{\circ} 116$. Two pulsation periods can be clearly identified (1950 and $2900 \mathrm{~s}$ ) and are more likely to be due to high-order non-radial $g$-mode oscillations than to radial or non-radial $p$-modes.
\end{abstract}

Key words. stars: chemically peculiar - stars: early-type - subdwarfs - stars: individual: LS IV $-14^{\circ} 116$ - stars: oscillations

\section{Introduction}

Pulsations in stars can arise when a local maximum in opacity is situated at an appropriate depth in the stellar atmosphere. Pulsations in subdwarf B (sdB) stars were first discovered by Kilkenny et al. (1997). They are thought to be driven by an opacity peak due to iron often referred to as the "Z-bump" (Charpinet et al. 1997). Such stars (EC14026 or V361 Hya variables) have pulsation periods of $\sim 100-200 \mathrm{~s}$ and amplitudes of $\sim 0.01 \mathrm{mag}$ (Kilkenny 2002). More recently, Green et al. (2003) have reported another class of pulsating sdB star with periods of around $1 \mathrm{~h}$ and amplitudes of $\lesssim 0.05 \mathrm{mag}$ (PG 1716+426 stars: Green et al. 2003). These pulsations were recently ascribed to non-radial $g$-modes with $l=3$ or 4 with very high radial wavenumbers (Fontaine et al. 2003). Such stars are generally cooler than the V361 Hya variables.

Helium-rich sdB (He-sdB) stars have similar effective temperatures $\left(T_{\text {eff }}\right)$ to normal sdB stars but have generally lower surface gravities ( $\log g$, Ahmad \& Jeffery 2003). They roughly straddle the top of the V361 Hya instability zone and lie close to the bottom of the $Z$-bump instability finger for extreme helium stars (Fig. 1). With less hydrogen, which tends to damp oscillations, a systematic search for variability in He-sdB stars was suggested by Jeffery \& Saio (1999). Results of an initial survey were inconclusive (Ahmad et al. 2004).

LS IV- $14^{\circ} 116$ was first listed in volume IV of the Luminous Stars in the Northern Milky Way catalogue (Nassau \& Stephenson 1963). Kilkenny \& Pauls (1990) later classified it as sdO from an optical spectrum. It was subsequently called sdO/He-rich (Viton et al. 1991) and was later included in a catalogue of helium-rich subdwarf B stars by Jeffery et al. (1996). Although the star shows significantly more helium $\left(n_{\mathrm{He}}=0.21\right.$, Ahmad et al. 2003) than other sdB stars, which

^ This paper uses observations made from the South African Astronomical Observatory (SAAO).

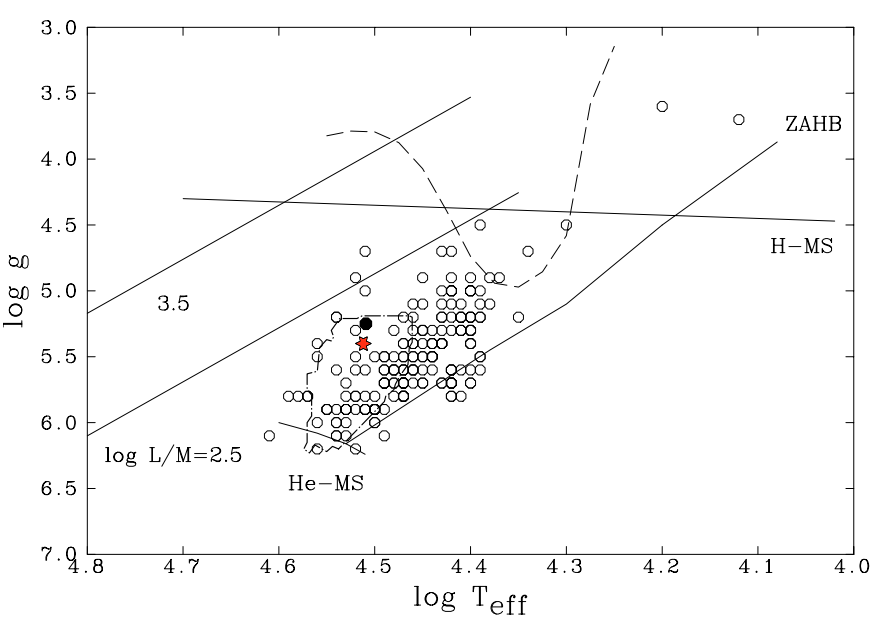

Fig. 1. Position of the He-sdB star $-\mathrm{LS} \mathrm{IV}-14^{\circ} 116$ (filled star) on the $\log g-T_{\text {eff }}$ diagram in comparison to PG $1605+072$ (filled circle) and other sdB stars (open circles). The dot-dash line around LS IV-14 116 represents the instability region where pulsating V361 Hya stars are found with $N \geq 5$ excited radial $(l=0)$ modes (Charpinet et al. 2001). The dashed line represents the instability boundary for lowmass $\left(0.5 M_{\odot}\right)$, extremely helium-rich $(X=0.00)$ stars (Jeffery \& Saio 1999).

generally show substantial depletions of helium $\left(0.001<n_{\mathrm{He}}<\right.$ 0.03 , Edelmann et al. 2003), LS IV $-14^{\circ} 116$ is not as extremely helium-rich as some other He-sdB stars (e.g. LB 1766, Kilkenny \& Busse 1992). This is evident from the presence of strong hydrogen Balmer lines (see Ahmad et al. 2003). Nevertheless it is fair to refer to all sdB stars showing significantly more helium than normal as "helium rich".

In this paper we report photometry of LS IV- $14^{\circ} 116$ obtained in 2004 May as part of our systematic monitoring programme (Ahmad et al. 2004). 
Table 1. Photometric observations of LS IV $-14^{\circ} 116$.

\begin{tabular}{ccccrr}
\hline \hline Night & Date & Run & UT Start & $t_{\text {exp }}$ & $N_{\text {obs }}$ \\
\hline N1 & 20040520 & 203 & $00: 30: 06$ & 8 & 1269 \\
N2 & 20040521 & 304 & $00: 23: 31$ & 11 & 238 \\
N3 & 20040522 & 403 & $00: 22: 42$ & 7 & 2080 \\
N4 & 20040524 & 605 & $00: 46: 43$ & 12 & 1065 \\
N5 & 20040525 & 703 & $00: 03: 14$ & 15 & 942 \\
\hline
\end{tabular}

Table 2. Stars present on the UCT CCD frame.

\begin{tabular}{|c|c|c|c|c|}
\hline Star & $\alpha_{J 2000}$ & $\delta_{J 2000}$ & GS2.2 identifier & $R$ mag \\
\hline \multicolumn{5}{|c|}{ LS IV-14ำ 116} \\
\hline & 205738.9 & -142542.2 & S3313303328 & 13.2 \\
\hline \multicolumn{5}{|c|}{ Comp 1} \\
\hline & 205741.4 & -142611.4 & S331330313746 & 14.1 \\
\hline \multicolumn{5}{|c|}{ Comp 2} \\
\hline & 205741.6 & -142623.7 & S331330313647 & 16.7 \\
\hline
\end{tabular}

\section{Observations and data reduction}

High-speed photometric observations of LS IV-14 116 were obtained with the Elizabeth Telescope $(1.0 \mathrm{~m})$ at the South African Astronomical Observatory (SAAO) on five separate nights in 2004 May as part of our follow-up observations of suspected pulsating He-sdB stars (Ahmad et al. 2004). LS IV $-14^{\circ} 116$ had earlier been monitored on two nights in 2003 April for $\sim 2000$ s, but no short-period oscillations had been detected. Hence the the star was not on the original list of follow-up targets. However, due to poor observing conditions at the telescope, $\mathrm{LS} \mathrm{IV}-14^{\circ} 116$ was selected for monitoring as it is relatively bright $(B \sim 13 \mathrm{mag}$ ). Hints of variability were present in the light curve obtained on the first night. Observations were therefore made on four subsequent nights.

The 2004 observations were made with University of Cape Town (UCT) high-speed CCD camera operated in "frame-transfer" mode. In this mode data are acquired continuously since there is essentially no dead time for the CCD readout.

Weather conditions were not photometric on nights two, four and five. The integration time was adjusted on each night to ensure high signal-to-noise $(S / N)$. No filter was used for the observations. A summary of the observations is shown in Table 1, which includes the run label, the start time, individual exposure time $t_{\exp }$ in seconds, and the number of observations $N_{\text {obs }}$ for each night.

The raw CCD frames were bias subtracted and flat-fielded using standard procedures. The processed frames were then reduced in real-time using D.O'Donoghue's DuPhot routines (Schechter et al. 1993) available at the telescope. The raw magnitudes were extracted by fitting a Point Spread Function (PSF) to each star. Due to the small size of the UCT CCD $\left(109^{\prime \prime} \times 74^{\prime \prime}\right)$, only two nearby comparison stars could be observed (Table 2). Comparison 2 is quite faint hence only comparison 1 was used for the differential correction. Comparison 2 was checked against comparison 1 for variability.

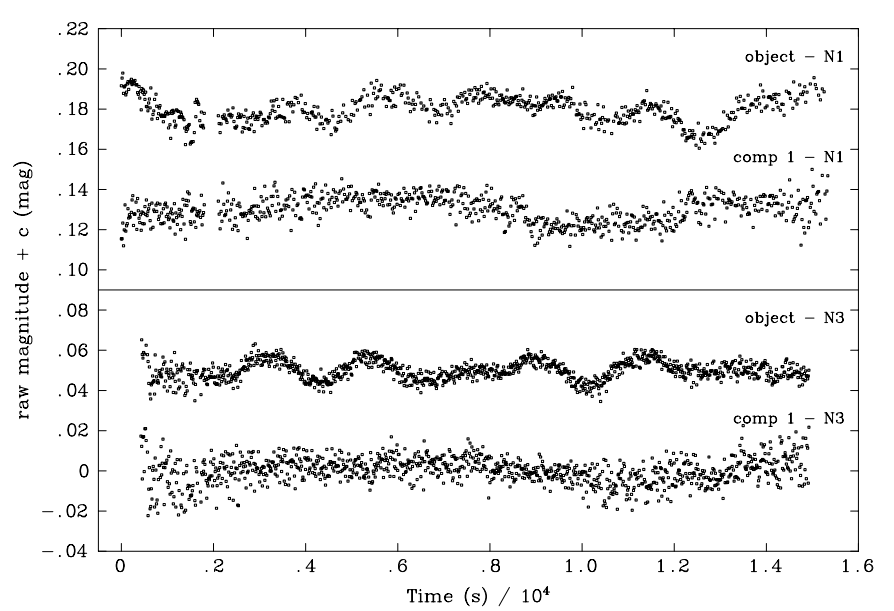

Fig. 2. Detrended nondifferential light curve of LS IV $-14^{\circ} 116$ and comparison 1 for nights one (N1) and three (N3).

Since the observing runs were quite long and no filter was used, the effects of atmospheric extinction are quite significant creating long term trends in the data. The data were detrended by dividing the reduced light curve from each individual night with a second order polynomial. Bad data points were removed from the final light curve. Detrended nondifferential light curve for two nights are shown in Fig. 2. The detrended and cleaned data from individual nights were then combined into a single light curve.

\section{Is LS IV $-14^{\circ} 116$ variable?}

The variability seen in LS IV $-14^{\circ} 116$ is intrinsically due to the star and not due to observing conditions, as Fig. 2 clearly shows short period variations $(\sim 1000 \mathrm{~s})$ in $\mathrm{LS}$ IV $-14^{\circ} 116$ which are not present in the light curve of the comparison. Longer term variations $(\sim 10000 \mathrm{~s})$ apparent in both stars may be due to transparency fluctuations.

We computed Scargle periodograms (Fig. 3) from the light curve (Fig. 2) of LS IV-14 116 and comparison 1 for nights one (N1) and three (N3). Periodograms were also computed for the combined data from the two nights $(\mathrm{N} 1+\mathrm{N} 3)$. If a period is present on individual nights and is still present in the combined data, then it is likely to be intrinsic to the object. Extrinsic factors (e.g. observing conditions) will normally, but not always, cancel when data from several nights are combined. In Fig. 3 we see that the frequency around $0.1 \mathrm{mHz}$ is most likely to be due to long term atmospheric trends which are not completely removed by detrending as it is seen in all data. However the periods around 0.35 and $0.50 \mathrm{mHz}$ are present only in the periodogram of $\mathrm{LS} \mathrm{IV}-14^{\circ} 116(\mathrm{~N} 1, \mathrm{~N} 3$ and $\mathrm{N} 1+\mathrm{N} 3)$ and $n o t$ in the periodogram of the comparison. Differentially corrected, detrended and cleaned data from all five nights were combined into a single light curve. This final light curve was used for the frequency analysis. The periods in the raw light curve of $\mathrm{LS}$ IV $-14^{\circ} 116(\mathrm{~N} 1, \mathrm{~N} 3$ and $\mathrm{N} 1+\mathrm{N} 3)$ also appear in the periodogram of the final light curve. 


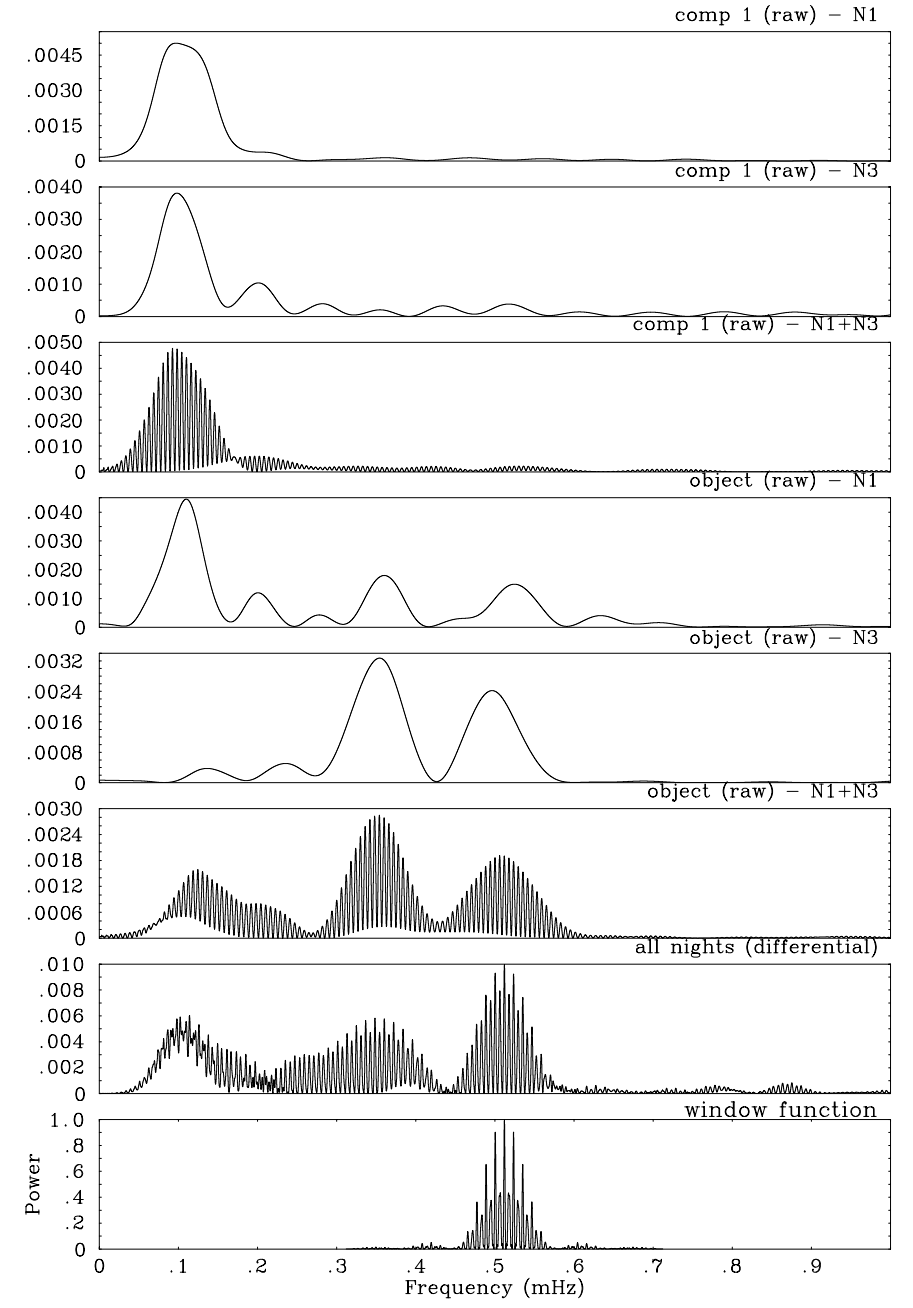

Fig. 3. Panels one to three show the Scargle periodogram of comparison 1 computed from the raw light curve for nights one $(\mathrm{N} 1)$ and three $(\mathrm{N} 3)$ and both nights combined $(\mathrm{N} 1+\mathrm{N} 3)$. Panels four to six show the periodogram for LS IV $-14^{\circ} 116$ for $\mathrm{N} 1, \mathrm{~N} 3$ and $\mathrm{N} 1+\mathrm{N} 3$. The second bottom panel shows the periodogram for the combined (differentially corrected) data from all five nights. The bottom panel shows the window function for the entire dataset shifted to the principal frequency.

\section{Frequency analysis}

The final light curve was subjected to a formal frequency analysis assuming that the variations seen in the data comprise a multi-periodic sinusoidal oscillation. The discrete Fourier transform (DFT, Deeming 1975) was constructed. Although the data are well-sampled locally, the window function is compli-

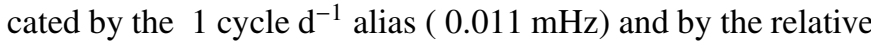
brevity of the time series (Fig. 3).

After selecting a number of frequencies from the DFT, a multi-parameter solution was obtained using a gradient expansion algorithm to compute a non-linear least squares fit in which the frequency, amplitude and phase of each independent sinusoid are free parameters. The resulting fit was subtracted from the original data and the DFT of the residual light curve was inspected.
Table 3. Results of frequency analysis as found while pre-whitening.

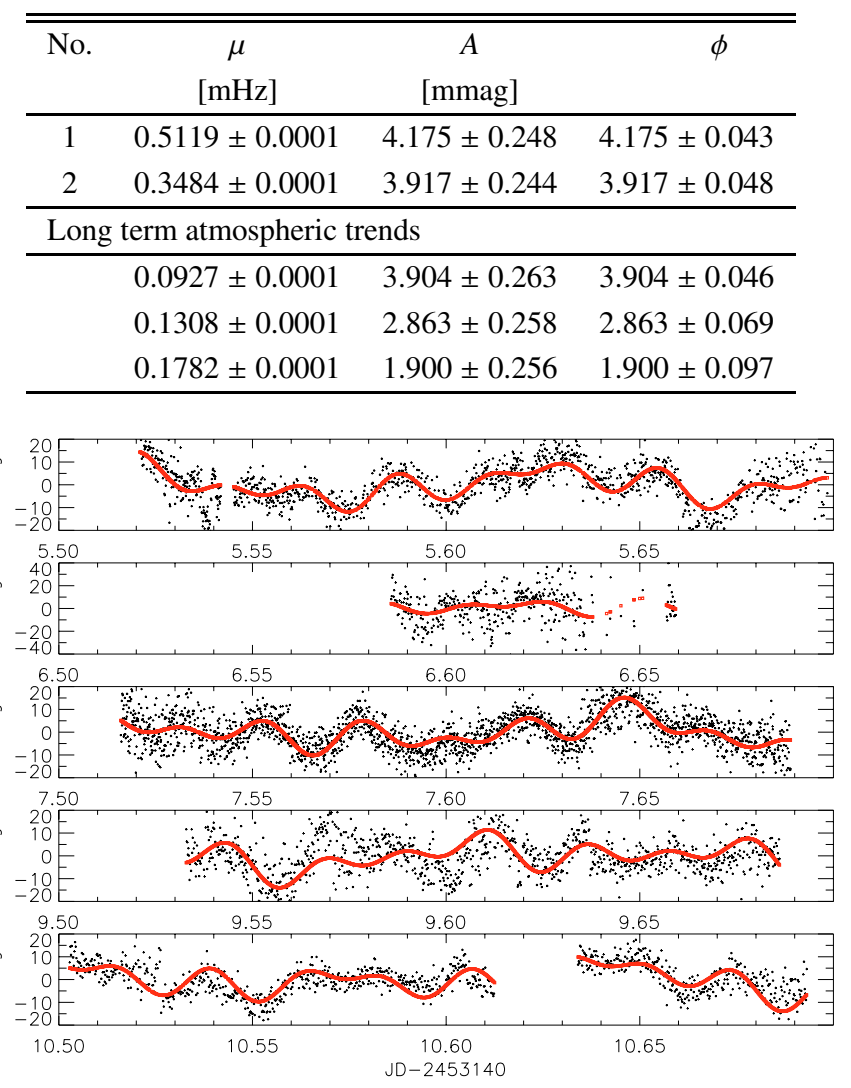

Fig. 4. Detrended differential light curve of LS IV $-14^{\circ} 116$ along with best fit model (solid line).

The multi-parameter solutions would, on occasion, find inappropriate solutions in which one or more frequencies were considerably different from those provided as initial estimates. Such solutions would be rejected. A criterion for a good solution was that the inclusion or omission of any given frequency would not significantly affect the frequencies or amplitude of any stronger components of the solution. The persistence of significant power after the removal of five frequencies points to the need for more accurate photometry with a much cleaner window function.

A short section of very noisy data obtained early on 2004 May 21 was omitted from the final analysis; its inclusion had no effect on the frequencies identified, but a small effect on the amplitudes measured. The final solution is listed in Table 3 and shown in Fig. 4. Three low frequencies (Table 3) are also included in the final solution shown in Fig. 4, these are also seen in the periodogram of the comparison star (Fig. 3) and are most likely due to residual long term atmospheric trends which are not totally corrected by detrending.

\section{Pulsations in LSIV $-14^{\circ} 116$}

LS IV $-14^{\circ} 116$ is shown on the $\log g-T_{\text {eff }}$ diagram in Fig. 1, using $T_{\text {eff }}=32500 \pm 700 \mathrm{~K}, \log g=5.4 \pm 0.1($ Ahmad \& Jeffery 2003). The contour around LS IV $-14^{\circ} 116$ marks the region of instability where most V361 Hya stars are found 
with typical periods of $\sim 100-250$ s (Charpinet et al. 2001). The periods seen in LS IV-14ำ 116 (1953-2870s, Table 3$)$ are very long, even in comparison with PG 1605+072 (295-573 s, Kilkenny et al. 1999) which lies very close to LS IV-14²116

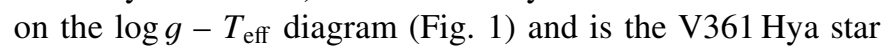
with the longest periods. However, they are much shorter than those seen in the non-radially pulsating low-mass helium-rich star HD 160641 (8.2-41 h, Lynas-Gray et al. 1987).

We can compare the pulsations in LS IV $-14^{\circ} 116$ with those in PG $1605+072$. If, for the sake of argument, the longest period seen in each star belongs to the fundamental radial mode, then we can compare their mean radii using the period-mean density relation (Cox 1980). Since we also know their surface gravities, we can eliminate mass to obtain $R \propto P^{2} g$. For LS IV $-14^{\circ} 116, P_{\mathrm{LS}}=2870$ and $\log g_{\mathrm{LS}}=5.4 \pm 0.1$. For $\mathrm{PG} 1605+072, P_{\mathrm{PG}}=573$ and $\log g_{\mathrm{PG}}=5.25 \pm 0.1$. Hence $R_{\mathrm{PG}} / R_{\mathrm{LS}}$ would be $\sim 0.03 \pm 0.01$ and, since $M \propto P^{4} g^{2}$, the corresponding mass ratio $\left(\sim 6 \pm 3 \times 10^{-5}\right)$ would be unphysical. Hence the assumption of oscillations in the fundamental radial mode is untenable. V361 Hya stars are primarily $p$-mode oscillators, including modes with $l=0$. With such long periods, it therefore seems likely that LS IV $-14^{\circ} 116$ shows nonradial $g$-modes, possibly as seen in the the cooler PG $1715+426$ stars. Fontaine et al. (2003) compute periods for $g$-modes in sdB stars; at the observed periods in LS IV $-14^{\circ} 116$, the radial order must be high, increasing from $k \sim 4-12$ at degree $l=0,1$ to $k>10$ for $l=3,4$. However, although excited in PG $1716+426$ stars, these modes are stable at $T_{\text {eff }} \gtrsim$ $25000 \mathrm{~K}$ (Fontaine et al. 2003). No other published pulsation studies successfully account for the variability observed in LS IV $-14^{\circ} 116$. Pulsation calculations including an appropriately helium-enriched envelope have still to be undertaken.

\section{Conclusions}

In the course of systematic monitoring of helium-rich subdwarf B stars, we have found the moderate He-sdB LS IV $-14^{\circ} 116$ to show multi-periodic photometric variability. On the basis of a comparison with the V361 Hya star PG 1605+072 we argue that the variability is due to non-radial $g$-mode oscillations of high radial order. Current theory suggests that such modes should be stable. These observations therefore offer a new challenge to stellar pulsation theory.

Meanwhile, with two modes clearly present, and more likely to be found in a sustained multi-site observing effort,
LS IV $-14^{\circ} 116$ offers many opportunities for further study. Ultimately, direct measurements of mass and radius will allow an informed discussion of its evolutionary origin and an explanation for its unusual surface helium abundance. In the longer term they may help to explain the connection, if any, between the normal and the helium-rich subdwarf B stars.

Acknowledgements. This research is supported by the UK Particle Physics and Astronomy Research Council through grants PPA/G/S/2002/00546 and PPA/G/O/2003/00044 and by the Northern Ireland Department of Culture, Arts and Leisure (DCAL). This research has made use of NASA's ADS. Contructive comments from two referees are appreciated.

\section{References}

Ahmad, A., \& Jeffery, C. S. 2003, A\&A, 402, 335

Ahmad, A., Jeffery, C. S., Solheim, J.-E., \& Østensen, R. 2004, Ap\&SS, 291, 435

Charpinet, S., Fontaine, G., Brassard, P., et al. 1997, ApJ, 483, L123

Charpinet, S., Fontaine, G., \& Brassard, P. 2001, PASP, 113, 775

Cox, J. P. 1980, Theory of Stellar Pulsation (Princeton: Princeton Univ. Press)

Deeming, T. J. 1975, Ap\&SS, 36, 137

Edelmann, H., Heber, U., Hagen, H.-J., et al. 2003, A\&A, 400, 939

Fontaine, G., Brassard, P., Charpinet, S., et al. 2003, ApJ, 597, 518

Green, E. M., Fontaine, G., Reed, M. D., et al. 2003, ApJ, 583, L31

Jeffery, C. S., \& Saio, H. 1999, MNRAS, 308, 221

Jeffery, C. S., Heber, U., Hill, P. W., et al. 1996, in Hydrogen Deficient Stars, ed. C. S. Jeffery, \& U. Heber, ASP Conf. Ser., 96, 471

Kilkenny, D. 2002, in Radial and Nonradial Pulsations as Probes of Stellar Physics, ed. C. Aerts, T. R. Bedding, \& J. Christensen-Dalsgaard, ASP Conf. Ser., 259, 356

Kilkenny, D., \& Pauls, L. 1990, MNRAS, 244, 133

Kilkenny, D., \& Busse, J. 1992, MNRAS, 258, 57

Kilkenny, D., Koen, C., O’Donoghue, D., \& Stobie, R. S. 1997, MNRAS, 285, 640

Kilkenny, D., Koen, C., O’Donoghue, D., et al. 1999, MNRAS, 303, 525

Lynas-Gray, A. E., Kilkenny, D., Skillen, I., \& Jeffery, C. S. 1987, MNRAS, 227, 1073

Nassau, J. J., \& Stephenson, C. B. 1963, Hamburger Sternw. Warner $\&$ Swasey Obs., 0

Schechter, P. L., Mateo, M., \& Saha, A. 1993, PASP, 105, 1342

Viton, M., Deleuil, M., Tobin, W., Prévot, L., \& Bouchet, P. 1991, A\&A, 242, 175 